\title{
THE SEVEN HABITS OF HIGHLY EFFECTIVE DRUG DISCOVERERS?
}

\author{
This month sees the further expansion of the front end of Nature Reviews Drug Discovery, \\ with the addition of two new regular features, to give a total of seven short, interdisciplinary, \\ monthly features aimed at keeping you 'in the know' across the spectrum of drug discovery.
}

A cartoon posted in our office has a picture of a scientist clearing out his lab, with two colleagues walking past outside. One says to the other, "Sad story; he was a great researcher but took a four-week vacation and never caught up on the literature again. "Keeping up with the field can be a daunting task, especially so when one considers the interdisciplinary nature of drug discovery and development.

Given that no one ever finds the leisure to do all the reading they would like to, it sometimes helps to have the key points encapsulated in concise form. For that reason, the front end of Nature Reviews Drug Discovery carries short articles that attempt to cover the gamut of our field, allowing readers to dip in and stay abreast of the news. Those that have followed the journal from its launch will have noticed a gradual growth in the number of these features, and April's issue introduces two new ones, the Patent Primer, acting as a guide to the often frustratingly complex world of intellectual property law, and An Audience With..., which brings you face to face with a leading light in drug discovery.

For those that aren't familiar with the layout, or those that would like refreshing, there are seven different article types at the beginning of each issue of the journal (spanning pages 287-300 in this issue). Six of these are contained within the News \& Analysis section, which opens with three pages of news stories that attempt to delve into the science behind recent high-profile events. Then follows Patentwatch, which reports on those worldwide patent rulings of greatest consequence for drug discovery and development. In response to the difficulties that all untrained people have in unravelling the complications of patent law, next comes our new Patent Primer, which will act as a step-by-step guide through the world frequented by such mysterious beasts as 'prior art', 'inventive steps', 'oppositions and appeals' and the 'doctrine of equivalents.' Taking an international view of the subject, these features will also highlight important country-specific differences in patent law, and although this month's article focuses on introducing the European patent process, next month's will focus on the requirements for patentability in the United States.

Our other new feature, An Audience With..., follows the Patent Primer. These articles will be used to pose key questions around single themes to leading figures in the drug discovery and development world — questions that many would no doubt love to ask if they could only get the chance. So, for instance, we begin the series (on page 292) by asking Mark Fishman, President of the Novartis Institutes for BioMedical Research, why someone of his background was appointed to this role, and how he will measure his effectiveness.

This latter question, of how to assess success in one's role, seems to be a particularly crucial one given the fact that people, in general, are spending less and less time in any one job. True measures of someone's impact are therefore becoming harder to come by, especially in an endeavour as drawn-out as the drug discovery and development processs. For instance, the timeline included in the news story about Mark McClellan's move from the FDA (on page 289) underlines the shortness of tenure of recent FDA commissioners, and raises the question of what performance measures can and should be applied to such a role.

The News \& Analysis section is capped off by a pair of two-page features: From the Analyst's Couch, which takes a look at the market dynamics surrounding a particular area of science, and Fresh from the Pipeline, which summarizes the science and likely market impact of exciting, recently approved drugs. Finally, the Highlight section, which has been with us from the start, points to some of the most interesting examples of interdisciplinary drug research in the current literature, with short stories summarizing their findings and impact. 